\title{
Generalized Function Projective Lag Synchronization in Fractional-Order Chaotic Systems
}

\author{
Yancheng Ma, Guoan Wu, and Lan Jiang
}

\begin{abstract}
In this paper, a new synchronization of fractionalorder chaotic systems called generalized function projective lag synchronization is introduced. This synchronization method is a generalization of function projective synchronization and function projective lag synchronization. Based on the stability theorem of linear fractional order systems, a suitable nonlinear fractional-order controller is designed for the synchronization of different structural systems. Three examples are given to verify the effectiveness of the proposed method.
\end{abstract}

Index Terms - Chaos, fractional order, generalized function projective lag synchronization, nonlinear controller.

\section{INTRODUCTION}

Since pioneering and meaningful work of Pecora and Carroll [1], chaotic synchronization has become a hot topic and been studied by more and more scholars in recent decades. It has many applications in the field of physics, chemistry, biology, and others especially in secure communication. From the existing literature, there are many different types of synchronization method, such as complete synchronization [2]-[5], projective synchronization [6], generalized synchronization [7], [8], robust synchronization [9], function projective synchronization (FPS) [10], function projective lag synchronization (FPLS) [11]. There are many control scheme utilizing in chaotic synchronization, such as adaptive control [12]-[14], sliding mode control [15]-[17], fuzzy sliding mode control [18], [19]. In [20], a reference system is added to make the synchronization more complex. Inspired by [20], we introduce a new type of synchronization method named generalized function projective lag synchronization (GFPLS) which is a generalization of FPS and FPLS.

The organization of this paper is as follows. The GFPLS method is introduced in Section II. Simulation and results are presented in Section III. In Section IV, conclusions are proposed.

\section{THE GFPLS METHOD}

\section{A. The Definition of GFPLS}

Choose fractional-order drive system as:

$$
\begin{aligned}
& D^{\varepsilon} x(t)=l(x(t)) \\
& x(t)=x(0), t \in[-\omega, 0]
\end{aligned}
$$

where $D^{\varepsilon}$ signifies the fractional-order differential operator.

Manuscript received March 28, 2016; revised September 20, 2016.

Authors are with the Huazhong University of Science and Technology, China (e-mail: TONYMA1989@qq.com). $\varepsilon \in(0,1)$ denotes the fractional order of drive system, $l: R^{c} \rightarrow R^{c}$ indicates the continuous function of drive system. $x(t)=\left(x_{1}(t), x_{2}(t), \cdots x_{c}(t)\right)^{T} \in R^{c}$ indicates the state vector of drive system, c denotes the dimension of drive system. $\omega>0$ is the time delay. The fractional-order response system is defined as:

$$
D^{\eta} y(t)=f(y(t))+u(x(t-\omega), y(t), z(t))
$$

where $D^{\eta}$ signifies the fractional-order differential operator. $\eta \in(0,1)$ denotes the fractional order of response system, $f: R^{d} \rightarrow R^{d} \quad$ indicates the continuous function. $y(t)=\left(y_{1}(t), y_{2}(t), \cdots y_{d}(t)\right)^{T} \in R^{d}$ indicates the state vector of response system, $\mathrm{d}$ denotes the dimension of response system. $u(t)=\left(u_{1}(t), u_{2}(t), \cdots u_{d}(t)\right)^{T} \in R^{d}$ is the nonlinear controller to be designed later. Choose fractional- order reference system as:

$$
D^{q} z(t)=h(z(t))
$$

where $D^{q}$ signifies the fractional-order differential operator. $q \in(0,1)$ indicates the fractional order of reference system, $h: R^{d} \rightarrow R^{d}$ denotes the continuous function. $z(t)=\left(z_{1}(t), z_{2}(t), \cdots z_{d}(t)\right)^{T} \in R^{d}$ denotes the state vector, System (3) is an attractor. Define error state vector as:

$$
e(t)=y(t)-C(x(t-\omega)) x(t-\omega)-z(t)
$$

where $C(x(t-\omega)) \in R^{d \times c}, x_{\omega}=x(t-\omega)$.

There exists two conditions, one condition is system dimension $d \leq c$

$$
C\left(x_{\omega}\right)=\left(\begin{array}{cc}
K\left(x_{\omega}\right) & 0 \\
0 & Q\left(x_{\omega}\right)
\end{array}\right)
$$

where the matrix

$$
\begin{gathered}
K\left(x_{\omega}\right)=\operatorname{diag}\left\{c_{1}\left(x_{\omega}\right), c_{2}\left(x_{\omega}\right), \cdots, c_{\tau-1}\left(x_{\omega}\right)\right\}, \\
\tau=\min \{c, d\} \text { and } Q\left(x_{\omega}\right)=\left(c_{\tau}\left(x_{\omega}\right), \cdots, c_{\mu}\left(x_{\omega}\right)\right),
\end{gathered}
$$

$\mu=\max \{c, d\} \quad, \quad$ the other condition is system dimension $d>c$, the matrix is defined as

$$
C\left(x_{\omega}\right)=\left(\begin{array}{cc}
K\left(x_{\omega}\right) & 0 \\
0 & Q^{T}\left(x_{\omega}\right)
\end{array}\right)
$$

Definition 1: GFPLS is realized. Assume there exists a nonlinear controller $u\left(x_{\omega}, y(t), z(t)\right)$ such that $\lim _{t \rightarrow \infty}\|e(t)\|=0$. 
Remark 1: When system (3) denotes constant zero, GFPLS degenerated to FPLS.

Remark 2: When system (3) denotes constant zero and time delay is zero, GFPLS degenerated to FPS.

Remark 3: System (3) can be other attractors, such as chaos, hyperchaos, periodic function, and quasi period function.

\section{B. The Stability Analysis of GFPLS}

Consider controller as:

$$
\begin{aligned}
& u=D^{\eta}\left(C\left(x_{\omega}\right) x_{\omega}\right)+D^{\eta}(z)-f\left(C\left(x_{\omega}\right) x_{\omega}\right) \\
& -f(z)+v+G\left(x_{\omega}, y(t), z(t)\right) e
\end{aligned}
$$

where $\quad v \in R^{\mathrm{d}} \quad$ is the compensation vector, $G\left(x_{\omega}, y(t), z(t)\right) \in R^{d \times d}$ is a polynomial matrix. According to (2), (3) and (5), we obtain the fractional-order error system as follows:

$$
D^{\eta} e(t)=\left(F\left(x_{\omega}, y(t), z(t)\right)+G\left(x_{\omega}, y(t), z(t)\right)\right) e(t)
$$

where $F\left(x_{\omega}, y(t), z(t)\right) e(t)=f(y(t))-f\left(C\left(x_{\omega}\right) x_{\omega}\right)-f(z(t))+v$.

Proposition 1: $C\left(x_{\omega}\right)$ and time delay is given, GFPLS is accomplished if there exists $G\left(x_{\omega}, y(t), z(t)\right) \in R^{d \times d}$ such that

$$
F\left(x_{\omega}, y(t), z(t)\right)+G\left(x_{\omega}, y(t), z(t)\right)=-N\left(x_{\omega}, y(t), z(t)\right)
$$

where $N=\left[n_{i, j}\right] \in R^{d \times d}, i \neq j, n_{i, j}=-n_{j, i}, i=j, n_{i, j} \in R^{+}$

Proof: Assume $\lambda$ is an arbitrary eigenvalue of the matrix $F\left(x_{\omega}, y(t), z(t)\right)+G\left(x_{\omega}, y(t), z(t)\right)$ and the related nonzero eigenvector denotes $\zeta$. We may get

$$
\left(F\left(x_{\omega}, y(t), z(t)\right)+G\left(x_{\omega}, y(t), z(t)\right)\right) \zeta=\lambda \zeta
$$

Multiplying the $\zeta^{H}$ at the left of (8), we have

$$
\zeta^{H}\left(F\left(x_{\omega}, y(t), z(t)\right)+G\left(x_{\omega}, y(t), z(t)\right)\right) \zeta=\lambda \zeta^{H} \zeta
$$

where $\mathrm{H}$ denotes conjugate transpose of a matrix, $\lambda^{*}$ also indicates an eigenvalue of the matrix $F\left(x_{\omega}, y(t), z(t)\right)+G\left(x_{\omega}, y(t), z(t)\right)$

Similarly, we have

$$
\zeta^{H}\left(F\left(x_{\omega}, y(t), z(t)\right)+G\left(x_{\omega}, y(t), z(t)\right)\right)^{H} \zeta=\lambda^{*} \zeta^{H} \zeta
$$

According to (9) and (10), we obtain

$$
\begin{aligned}
& \lambda+\lambda^{*}=\zeta^{H}\left[\left(F\left(x_{\omega}, y, z\right)+G\left(x_{\omega}, y, z\right)\right)+\right. \\
& \left.\left(F\left(x_{\omega}, y, z\right)+G\left(x_{\omega}, y, z\right)\right)^{H}\right] \zeta / \zeta^{H} \zeta \\
& =-\zeta^{H} \psi \zeta / \zeta^{H} \zeta
\end{aligned}
$$

where $\zeta^{H} \zeta>0, \psi=\left(N\left(x_{\omega}, y, z\right)+N\left(x_{\omega}, y, z\right)^{H}\right) \in R^{d \times d}$.

$\mathrm{We}$ are able to obtain that $\psi$ denotes a real positivedefinite diagonal matrix. Thus, $\zeta^{H} \psi \zeta>0$, we can have

$$
\lambda+\lambda^{*}=2 \operatorname{real}(\lambda)<0
$$

Hence, we can obtain

$$
|\arg (\lambda)|>\pi / 2>\eta \pi / 2
$$

Based on the fractional-order stability theorem proposed in [21], the error system (6) asymptotically stabilizes at origin. GFPLS is achieved. The proof is completed.

\section{Simulation AND Results}

In this section, three examples presented in [11] are utilized to demonstrate the effectiveness of the GFPLS with the same and different dimension. A predictor-corrector method proposed in [22], [23] is used to solve fractionalorder differential equations.

\section{A. GFPLS with Same Dimension $d=c$}

Choose the fractional-order Rössler system as drive system.

$$
\begin{aligned}
& D^{\varepsilon} x_{1}(t)=-x_{2}(t)-x_{3}(t) \\
& D^{\varepsilon} x_{2}(t)=x_{1}(t)+\alpha_{1} x_{2}(t) \\
& D^{\varepsilon} x_{3}(t)=\alpha_{2}+x_{1}(t) x_{3}(t)-\alpha_{3} x_{3}(t) \\
& x(t)=x(0), t \in[-\omega, 0]
\end{aligned}
$$

When $\varepsilon=0.95,\left(\alpha_{1}, \alpha_{2}, \alpha_{3}\right)=(0.4,0.2,10), x(0)=(0.5,0.5,0.5)^{T}$, drive system shows chaotic attractor drawn in Fig. 1.

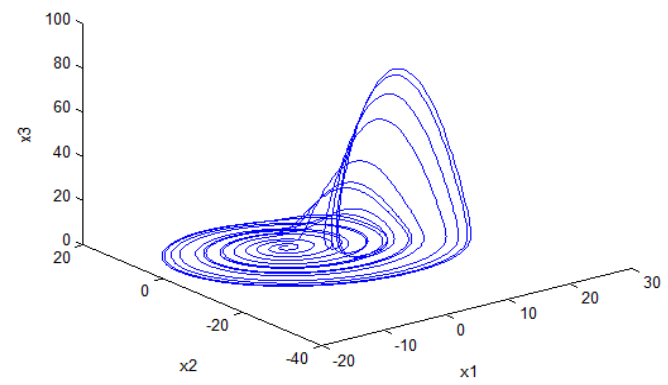

Fig. 1. The phase trajectory of the fractional-order Rössler system.

Choose the fractional-order Lü system as response system.

$$
\begin{aligned}
& D^{\eta} y_{1}(t)=\beta_{1}\left(y_{2}(t)-y_{1}(t)\right)+u_{1}\left(x_{\omega}, y(t), z(t)\right) \\
& D^{\eta} y_{2}(t)=-y_{1}(t) y_{3}(t)+\beta_{2} y_{2}(t)+u_{2}\left(x_{\omega}, y(t), z(t)\right) \\
& D^{\eta} y_{3}(t)=y_{1}(t) y_{2}(t)-\beta_{3} y_{3}(t)+u_{3}\left(x_{\omega}, y(t), z(t)\right)
\end{aligned}
$$

When $\eta=0.9,\left(\beta_{1}, \beta_{2}, \beta_{3}\right)=(35,28,3), \quad y(0)=(4.2,3.5,11)^{T}$, response system denotes chaotic attractor shown in Fig. 2.

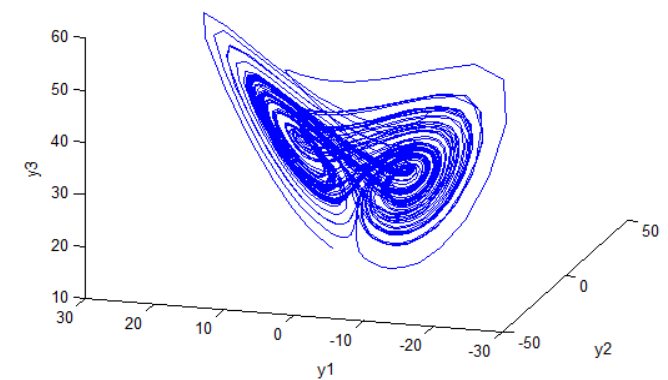

Fig. 2. The phase trajectory of the fractional-order Lü system.

Choose the fractional-order Chen system as reference system.

$$
\begin{aligned}
& D^{q} z_{1}(t)=\gamma_{1}\left(z_{2}(t)-z_{1}(t)\right) \\
& D^{q} z_{2}(t)=\left(\gamma_{3}-\gamma_{1}\right) z_{1}(t)-z_{1}(t) z_{3}(t)+\gamma_{3} z_{2}(t) \\
& D^{q} z_{3}(t)=z_{1}(t) z_{2}(t)-\gamma_{2} z_{3}(t)
\end{aligned}
$$

When $q=0.95,\left(\gamma_{1}, \gamma_{2}, \gamma_{3}\right)=(35,3,28), \quad z(0)=(15,12,31)^{T}$, reference system indicates chaotic attractor drawn in Fig. 3. 


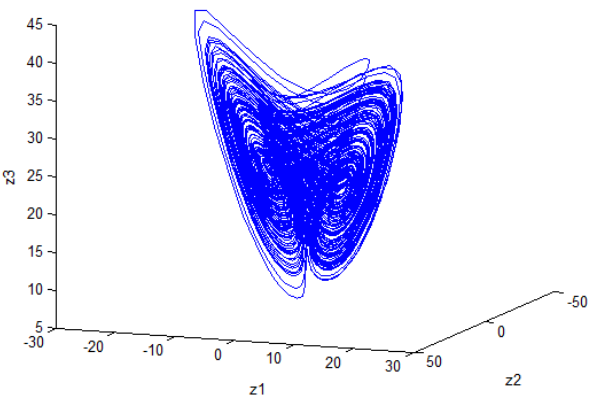

Fig. 3. The phase trajectory of the fractional-order Chen system.

The error states are defined as

$$
e_{i}(t)=y_{i}(t)-c_{i}\left(x_{\omega}\right) x_{i \omega}-z_{i}(t), x_{i \omega}=x_{i}(t-\omega),
$$

$i=1,2,3$. According to (5) and (15), we can get

$$
\begin{gathered}
F\left(x_{\omega}, y(t), z(t)\right)=\left(\begin{array}{ccc}
-\beta_{1} & \beta_{1} & 0 \\
-\left(c_{3}\left(x_{\omega}\right) x_{3 \omega}+z_{3}(t)\right) & \beta_{2} & -y_{1}(t) \\
y_{2}(t) & \left(c_{1}\left(x_{\omega}\right) x_{1 \omega}+z_{1}(t)\right) & -\beta_{3}
\end{array}\right) \\
G\left(x_{\omega}, y(t), z(t)\right)=\left(\begin{array}{ccc}
0 & \left(c_{3}\left(x_{\omega}\right) x_{3 \omega}+z_{3}(t)-\beta_{1}\right) & -y_{2}(t) \\
0 & -\beta_{2}-d_{1} & y_{1}(t) \\
0 & -\left(c_{1}\left(x_{\omega}\right) x_{1 \omega}+z_{1}(t)\right) & 0
\end{array}\right)
\end{gathered}
$$

where $d_{1}>0$, we can obtain

$$
F\left(x_{\omega}, y(t), z(t)\right)+G\left(x_{\omega}, y(t), z(t)\right)=-N\left(x_{\omega}, y(t), z(t)\right)
$$

where $N=\left[n_{i, j}\right] \in R^{d \times d}, \quad i \neq j, n_{i, j}=-n_{j, i}, \quad i=j, n_{i, j} \in R^{+}$, $i, j=1,2,3$. .

The GFPLS with same dimension is realized based on proposition 1.

When $\quad C\left(x_{\omega}\right)=\operatorname{diag}\left\{10 x_{2 \omega}+35, x_{3 \omega}-12.5,4 x_{3 \omega}-20 x_{1 \omega}\right\} \quad$, $\omega=0.5, d_{1}=5$, the error state curves of GFPLS with same dimension are shown in Fig. 4.

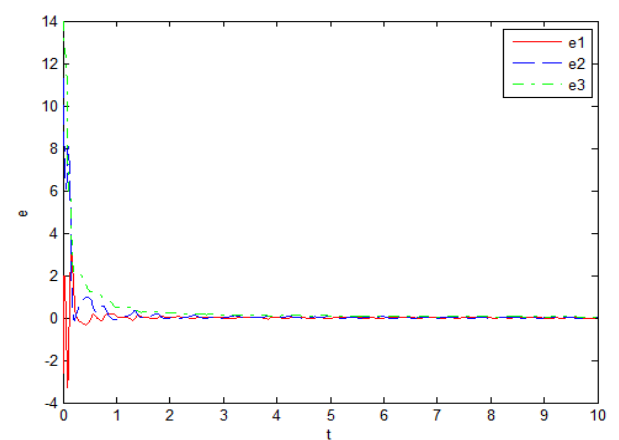

Fig. 4. The error state curves of GFPLS with same dimension.

\section{B. GFPLS with Different Dimension $d<c$}

Choose the fractional-order hyperchaotic Lorenz system as drive system

$$
\begin{aligned}
& D^{\varepsilon} x_{1}(t)=\alpha_{1}\left(x_{2}(t)-x_{1}(t)\right)+x_{4}(t) \\
& D^{\varepsilon} x_{2}(t)=\alpha_{2} x_{1}(t)-x_{2}(t)-x_{1}(t) x_{3}(t) \\
& D^{\varepsilon} x_{3}(t)=x_{1}(t) x_{2}(t)-\alpha_{3} x_{3}(t) \\
& D^{\varepsilon} x_{4}(t)=-x_{2}(t) x_{3}(t)-\alpha_{4} x_{4}(t) \\
& x(t)=x(0), t \in[-\omega, 0]
\end{aligned}
$$

When $\quad \varepsilon=0.98,\left(\alpha_{1}, \alpha_{2}, \alpha_{3}, \alpha_{4}\right)=(10,28,8 / 3,1)$ $x(0)=(-2.2,-6,8.3,-9)^{T} \quad$, drive system denotes hyperchaotic attractor shown in Fig. 5.
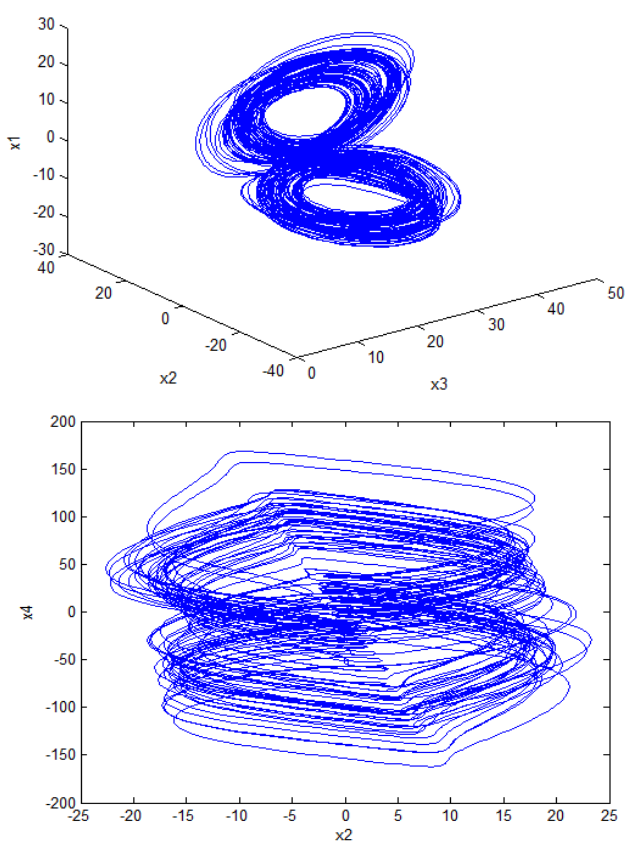

Fig. 5. The phase trajectory of the fractional-order hyperchaotic Lorenz system.

Choose the fractional-order Rössler system as response system.

$$
\begin{aligned}
& D^{\eta} y_{1}(t)=-y_{2}(t)-y_{3}(t)+u_{1}\left(x_{\omega}, y(t), z(t)\right) \\
& D^{\eta} y_{2}(t)=y_{1}(t)+\beta_{1} y_{2}(t)+u_{2}\left(x_{\omega}, y(t), z(t)\right) \\
& D^{\eta} y_{3}(t)=\beta_{2}+y_{1}(t) y_{3}(t)-\beta_{3} y_{3}(t)+u_{3}\left(x_{\omega}, y(t), z(t)\right)
\end{aligned}
$$

Choose the fractional-order Lü system as reference system.

$$
\begin{aligned}
& D^{q} z_{1}(t)=\gamma_{1}\left(z_{2}(t)-z_{1}(t)\right) \\
& D^{q} z_{2}(t)=-z_{1}(t) z_{3}(t)+\gamma_{2} z_{2}(t) \\
& D^{q} z_{3}(t)=z_{1}(t) z_{2}(t)-\gamma_{3} z_{3}(t)
\end{aligned}
$$

Based on (4), we can obtain the error states

$$
\begin{aligned}
& e_{1}(t)=y_{1}(t)-c_{1}\left(x_{\omega}\right) x_{1 \omega}-z_{1}(t) \\
& e_{2}(t)=y_{2}(t)-c_{2}\left(x_{\omega}\right) x_{2 \omega}-z_{2}(t) \\
& e_{3}(t)=y_{3}(t)-c_{3}\left(x_{\omega}\right) x_{3 \omega}-c_{4}\left(x_{\omega}\right) x_{4 \omega}-z_{3}(t)
\end{aligned}
$$

where $x_{i \omega}=x_{i}(t-\omega), i=1,2,3,4$. According to (5) and (18), we can have

$$
\begin{gathered}
F\left(x_{\omega}, y(t), z(t)\right)=\left(\begin{array}{ccc}
0 & -1 & -1 \\
1 & \beta_{1} & 0 \\
c_{3}\left(x_{\omega}\right) x_{3 \omega}+c_{4}\left(x_{\omega}\right) x_{4 \omega}+z_{3}(t) & 0 & y_{1}(t)-\beta_{3}
\end{array}\right) \\
G\left(x_{\omega}, y(t), z(t)\right)=\left(\begin{array}{ccc}
-d_{2} & 0 & L \\
0 & -\beta_{1}-d_{3} & -y_{2}(t) \\
0 & y_{2}(t) & -y_{1}(t)+\beta_{3}-d_{4}
\end{array}\right)
\end{gathered}
$$

where $L=1-c_{3}\left(x_{\omega}\right) x_{3 \omega}-c_{4}\left(x_{\omega}\right) x_{4 \omega}-z_{3}(t), d_{2}>0, d_{3}>0, d_{4}>0$, we can obtain

$$
F\left(x_{\omega}, y(t), z(t)\right)+G\left(x_{\omega}, y(t), z(t)\right)=-N\left(x_{\omega}, y(t), z(t)\right)
$$

where $N=\left[n_{i, j}\right] \in R^{d \times d}, i \neq j, n_{i, j}=-n_{j, i}, i=j, n_{i, j} \in R^{+}$, $i, j=1,2,3$.

Hence, the GFPLS with different dimension $(d<c)$ is achieved based on proposition 1 .

When $\omega=0.2, d_{2}=1, d_{3}=6, d_{4}=3$, 


$$
C\left(x_{\omega}\right)=\left(\begin{array}{llll}
-x_{2 \omega} / 2 & & & \\
& x_{2 \omega}+x_{3 \omega} & & \\
& & 1 & x_{1 \omega} / 2
\end{array}\right) .
$$

The error state curves of GFPLS with different dimension $(d<c)$ are shown in Fig. 6.

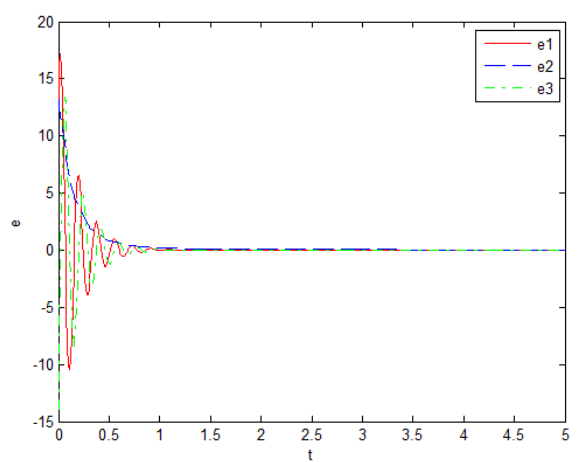

Fig. 6. The error state curves of GFPLS with different dimension $(\mathrm{d}<\mathrm{c})$.

\section{GFPLS with Different Dimension $d>c$}

Choose the fractional-order Lü system as drive system.

$$
\begin{aligned}
& D^{\varepsilon} x_{1}(t)=\alpha_{1}\left(x_{2}(t)-x_{1}(t)\right) \\
& D^{\varepsilon} x_{2}(t)=-x_{1}(t) x_{3}(t)+\alpha_{2} x_{2}(t) \\
& D^{\varepsilon} x_{3}(t)=x_{1}(t) x_{2}(t)-\alpha_{3} x_{3}(t) \\
& x(t)=x(0), t \in[-\omega, 0]
\end{aligned}
$$

Choose the fractional-order hyperchaotic Chen system as response system.

$$
\begin{aligned}
& D^{\eta} y_{1}(t)=\beta_{1}\left(y_{2}(t)-y_{1}(t)\right)+y_{4}(t)+u_{1}\left(x_{\omega}, y(t), z(t)\right) \\
& D^{\eta} y_{2}(t)=\beta_{2} y_{1}(t)-y_{1}(t) y_{3}(t)+\beta_{3} y_{2}(t)+u_{2}\left(x_{\omega}, y(t), z(t)\right) \\
& D^{\eta} y_{3}(t)=y_{1}(t) y_{2}(t)-\beta_{4} y_{3}(t)+u_{3}\left(x_{\omega}, y(t), z(t)\right) \\
& D^{\eta} y_{4}(t)=y_{2}(t) y_{3}(t)+\beta_{5} y_{4}(t)+u_{4}\left(x_{\omega}, y(t), z(t)\right)
\end{aligned}
$$

$$
\text { When } \quad \eta=0.96,\left(\beta_{1}, \beta_{2}, \beta_{3}, \beta_{4}, \beta_{5}\right)=(35,7,12,3,0.5)
$$

$y(0)=(1.2,2.1,3.1,0.1)^{T} \quad$ response system indicates the hyperchaotic attractor shown in Fig. 7.
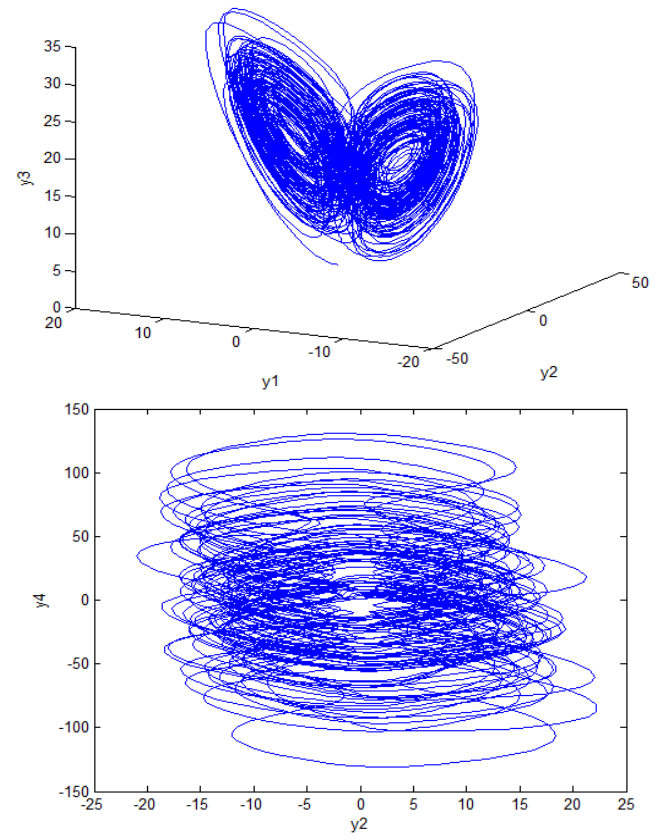

Fig. 7. The phase trajectory of the fractional-order hyperchaotic Chen system.
Choose the fractional-order hyperchaotic Lorenz system as reference system

$$
\begin{aligned}
& D^{q} z_{1}(t)=\gamma_{1}\left(z_{2}(t)-z_{1}(t)\right)+z_{4}(t) \\
& D^{q} z_{2}(t)=\gamma_{2} z_{1}(t)-z_{2}(t)-z_{1}(t) z_{3}(t) \\
& D^{q} z_{3}(t)=z_{1}(t) z_{2}(t)-\gamma_{3} z_{3}(t) \\
& D^{q} z_{4}(t)=-z_{2}(t) z_{3}(t)-\gamma_{4} z_{4}(t)
\end{aligned}
$$

According to (4), we can have the error states

$$
\begin{aligned}
& e_{1}(t)=y_{1}(t)-c_{1}\left(x_{\omega}\right) x_{1 \omega}-z_{1}(t) \\
& e_{2}(t)=y_{2}(t)-c_{2}\left(x_{\omega}\right) x_{2 \omega}-z_{2}(t) \\
& e_{3}(t)=y_{3}(t)-c_{3}\left(x_{\omega}\right) x_{3 \omega}-z_{3}(t) \\
& e_{4}(t)=y_{4}(t)-c_{4}\left(x_{\omega}\right) x_{3 \omega}-z_{4}(t)
\end{aligned}
$$

where $x_{i \omega}=x_{i}(t-\omega), \quad i=1,2,3$. According to (5) and (21), we can have

$$
\begin{gathered}
F\left(x_{\omega}, y(t), z(t)\right)=\left(\begin{array}{cccc}
-\beta_{1} & \beta_{1} & 0 & 1 \\
\beta_{2}-y_{3}(t) & \beta_{3} & -\left(c_{1}\left(x_{\omega}\right) x_{1 \omega}+z_{1}(t)\right) & 0 \\
c_{2}\left(x_{\omega}\right) x_{2 \omega}+z_{2}(t) & y_{1}(t) & -\beta_{4} & 0 \\
0 & y_{3}(t) & c_{2}\left(x_{\omega}\right) x_{2 \omega}+z_{2}(t) & \beta_{5}
\end{array}\right) \\
G\left(x_{\omega}, y(t), z(t)\right)=\left(\begin{array}{cccc}
d_{5} & -\beta_{1} & -\left(c_{2}\left(x_{\omega}\right) x_{2 \omega}+z_{2}(t)\right) & 0 \\
L_{1} & -\beta_{3}-d_{6} & c_{1}\left(x_{\omega}\right) x_{1 \omega}+z_{1}(t) & -y_{3}(t) \\
0 & -y_{1}(t) & 0 & 0 \\
-1 & 0 & -\left(c_{2}\left(x_{\omega}\right) x_{2 \omega}+z_{2}(t)\right) & -\beta_{5}-d_{7}
\end{array}\right)
\end{gathered}
$$

where $L_{1}=y_{3}(t)-\beta_{2}, 0<d_{5}<\beta_{1}, d_{6}>0, d_{7}>0$, we can obtain

$$
F\left(x_{\omega}, y(t), z(t)\right)+G\left(x_{\omega}, y(t), z(t)\right)=-N\left(x_{\omega}, y(t), z(t)\right)
$$

Where $N=\left[n_{i, j}\right] \in R^{d \times d}, i \neq j, n_{i, j}=-n_{j, i}, i=j, n_{i, j} \in R^{+}$, $i, j=1,2,3,4$.

Hence, the GFPLS with different dimension $(d>c)$ is realized based on proposition 1 .

When $\omega=0.05, d_{5}=25, d_{6}=5, d_{7}=4$,

$$
C\left(x_{\omega}\right)=\left(\begin{array}{ccc}
x_{2 \omega} & & \\
& x_{1 \omega}-x_{3 \omega} & \\
& & x_{1 \omega} / 2 \\
& & 2.5-x_{2 \omega}
\end{array}\right)
$$

The error state curves of GFPLS with different dimension $(d>c)$ are shown in Fig. 8.

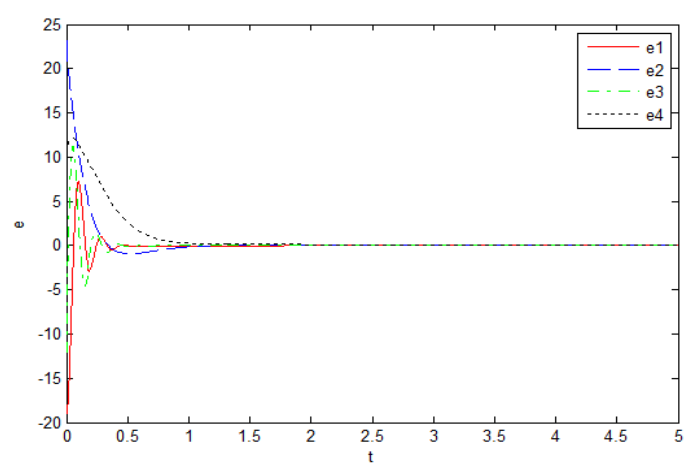

Fig. 8. The error state curves of GFPLS with different dimension $(\mathrm{d}>\mathrm{c})$.

\section{CONCLUSIONS}

In this paper, the GFPLS of fractional-order chaotic system is investigated based on the fractional-order stability theorem. This synchronization scheme is more complex and 
generalized than the existing synchronization method. The numerical simulations with the same and different dimension are given to illustrate the effectiveness of the proposed method. In the future, GFPLS has more potential to be applied in the secure communication.

\section{ACKNOWLEDGMENT}

I appreciate my supervisor Guoan $\mathrm{Wu}$ who gives me many opportunities and thank for every classmate in the institute of microwave technology and application. I also thank all the scholars who contribute to the improvement of the chaotic synchronization.

\section{REFERENCES}

[1] L. M. Pecora and T. L. Carroll, "Sychronizaiton in chaotic systems," Phys. Rev. Lett., vol. 64, pp. 35-38, Feb. 1990.

[2] J. G. Lu, "Chaotic dynamics of the fractional-order Ikeda delay system and its synchronization," Chine. Phys., vol. 15, pp. 301-305, 2006.

[3] C. P. Li, W. H. Deng, and D. Xu, "Chaos synchronization of the Chua system with a fractional order," Physica A, vol. 360, pp. 171-185, Feb. 2006.

[4] X. J. Wu, J. Li, and G. R. Chen, "Chaos in the fractional order unified system and its synchronization," J. Franklin Inst., vol. 345, pp. 392 401, July 2008.

[5] H. Taghvafard and G. H. Eqaee, "Phase and anti-phase synchronization of fractional order chaotic systems via active control," Commun. Nonlinear Sci. Numer. Simulat., vol. 16, pp. 40794088, Oct. 2011.

[6] X. Y. Wang and Y. J. He, "Projective synchronization of fractional order chaotic system based on linear separation," Phys. Lett. A, vol. 372, pp. 435-441, Jan. 2008.

[7] T. S. Wang and X. Y. Wang, "Generalized synchronization of fractional order hyperchaotic lorenz system," Mod. Phys. Lett. B, vol. 23, pp. 2167-2178, July 2009.

[8] R Zhou, X. R Cheng, and N. Y. Zhang, "Generalized synchronization between different fractional-order chaotic systems," Commun. Theor. Phys., vol. 50, pp. 931-934, 2008.

[9] K. Suwat, "Robust synchronization of fractional-order unified chaotic systems via linear control," Comput. Math. Appl., vol. 17, pp. 2670 2681, 2012.

[10] P. Zhou and W. Zhu "Function projective synchronization for fractional-order chaotic systems," Nonlinear Anal. Real World Appl., vol. 12, pp. 811-816, 2011.

[11] W. Sha, Y. Y. Guang, and W. Hu, "Function projective lag synchronization of fractional-order chaotic systems," Chin. Phys. B, vol. 23, pp. 1-7, 2014.

[12] R. A. Tang, Y. L. Liu, and J. K. Xue, "An extended active control for chaos synchronization," Phys. Lett. A, vol. 373, pp. 1449-1454, 2009.

[13] L.-X. Yang and J. Jiang, "Adaptive synchronization of drive-response fractional-order complex dynamical networks with uncertain parameters," Commun. Nonlinear Sci. Numer. Simulat., vol. 19, pp 1496-1506, 2014.
[14] S. Wen, Z. Zeng, and T. Huang, "Adaptive synchronization of memristor-based Chua's circuits," Phys. Lett. A, vol. 376, pp. 27752780, 2012.

[15] C. Yin, S. Dadras, and S. M. Zhong, "LMI based design of a sliding mode controller for a class of uncertain fractional-order nonlinear systems," J. Franklin Inst., vol. 349, pp. 3078-3101, 2012.

[16] S. H. Hosseinnia, R. Ghaderi, A. N. Ranjbar, M. Mahmoudian, and S. Momani, "Sliding mode synchronization of an uncertain fractional order chaotic system," Computers and Mathematics with Application, vol. 59, pp. 1637-1643, March 2010.

[17] P. Balasubramaniam, "Theoretical and practical applications of fuzzy fractional integral sliding mode control for fractional-order dynamical system," Nonlinear Dyn., vol. 80, pp. 249-267, April 2015.

[18] T.-C. Lin and T.-Y. Lee, "Chaos synchronization of uncertain fractional-order chaotic systems with time delay based on adaptive fuzzy sliding mode control," IEEE Tran. Fuzzy Sys., vol. 19, pp. 623635, Aug. 2011.

[19] B. Zhang and H. Guo, "Universal function projective lag synchronization of chaotic systems with uncertainty by using active sliding mode and fuzzy sliding mode control," Nonlinear Dyn., vol. 81, pp. 867-879, July 2015.

[20] B. Zhang and H. Li, "Universal projective synchronization of two different hyperchaotic systems with unknown parameters," Journal of Applied Mathematics, vol. 5, pp. 101-110, March 2014.

[21] D. Matignon, "Stability results of fractional differential equations with applications to control processing," Computational Engineering in Systems Applications, vol. 8, pp. 196-202, 1996.

[22] K. Diethelm, N. J. Ford, and A. D. Freed, "A predictor-corrector approach for the numerical solution of fractional differential equations," Nonlinear Dyn., vol. 29, pp. 3-22, July 2002.

[23] K. Diethelm, N. J. Ford, and A. D. Freed, "Detailed error analysis for a fractional adams method," Numer. Algorithms, vol. 36, pp. 31-52, May 2004

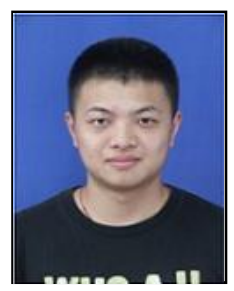

Yancheng Ma is a doctoral candidate which comes from the School of Optical and Electronic Information, Huazhong University of Science and Technology, Wuhan 430074, P. R. China. He was born in Jan. 16, 1989. Yancheng Ma has published two articles which are indexed by EI and SCI.

His research interests are artificial intelligence, chaos synchronization, optical communication.

Guoan Wu is a professor in the School of Optical and Electronic Information, Huazhong University of Science and Technology, Wuhan 430074 , P. R. China. Guoan Wu's research interests are artificial intelligence, microwave technology.

Lan Jiang is a teaching assistant in Wuhan Railway Vocational College of Technology. Her research interests are intelligent control and chaos synchronization. 